\title{
Impedance Controlled Twisted String Actuators for Tensegrity Robots
}

\author{
In-Won Park ${ }^{1}$ and Vytas SunSpiral ${ }^{2 *}$ \\ ${ }^{1}$ Oak Ridge Associated Universities, within the Intelligent Robotics Group, \\ NASA Ames Research Center, Moffett Field, CA, USA (in.w.park@ nasa.gov) \\ ${ }^{2}$ SGT Inc., within the Intelligent Robotics Group, \\ NASA Ames Research Center, Moffett Field, CA, USA (vytas.sunspiral@ nasa.gov) * Corresponding author
}

\begin{abstract}
We are developing impedance controlled twisted string actuators (TSA) for use in tensegrity robots, as an alternative to traditional spooled cable actuation. Tensegrity robots are composed of continuous tension and discontinuous compression elements, with no rigid joints between elements, which give them unique force distribution properties. The use of tensegrity robots is strongly motivated by biological examples, and they are capable of locomotion and manipulation by changing lengths of their continuous network of tensional elements, which is also the primary pathways for load transfer through the structure. TSA show the potential to address some of the unique engineering challenges faced by tensegrity structures, and provide unique qualities well suited to an actively controlled tension system, such as compact, light-weight mechanical structures, inherent compliance, variable "gearing", and the ability to transmit high forces with a very low input torque. The inherent variable compliance of impedance control is essential for tensegrity robots to move through and manipulate the environment, and is a natural match to the unique qualities of TSA. This paper briefly introduces the tensegrity robots in the NASA Ames Intelligent Robotics Group and an overview of their future application to space planetary exploration. Then the effectiveness and robustness of TSA are verified through the performance of impedance control modes.
\end{abstract}

Keywords: Tensegrity robots, twisted string actuator, impedance control.

\section{INTRODUCTION}

Over the last couple decades, there has been a lot of interest in tensegrity structures for use in space, especially as light-weight deployable mechanisms such as large aperture deployable antennas and expandable masts [1]. More recently, advances in computational power and control theory have enabled the nascent exploration of tensegrity robots for exploration purposes [2][5]. Tensegrity structures are composed of axially loaded compression elements suspended within a network of tension elements, which give them unique force distribution properties making them well suited for locomotion and manipulation tasks. Also, because of their structural simplicity as pure tension or compression elements, they can be light-weight, deployable, and have high strength to weight ratios. Yet with all their potential, there are still many engineering and control challenges to be overcome before these uniquely compliant robots can be practically deployed.

The most common method of actuating tensegrity robots for locomotion or manipulation tasks is to actively control the tension of the strings, which works linearly along the load paths. Due to their continuous tension network, tensegrity robots can control their structural stiffness by varying the pre-tension in their strings. Yet even under maximal pretension, tensegrity structures always maintain some amount of compliance, which is a desirable property when interacting with the environment (most modern manipulation and walking robots are incorporating compliance to deal with environmental in- teractions). Thus, tensegrity robots make an ideal target application for inherently compliant actuators. To date, the majority of tensegrity robots have been actuated by using traditional spooled cable actuation. Such an approach uses intermediate mechanisms such as gearboxes and spools which add weight and inefficiencies to the mechanism. In addition, a common failure mode occurs when the string goes slack and falls off the spool and wraps around the motor axis. To cope with these problems, we are investigating the use of twisted string actuators (TSA) that provide a simple, compact, light-weight, inherently compliant, mechanical approach to control the tension of the strings.

TSA is comprised of twisting strings where a pair (or more) of strings that are directly connected to an actuator acts as a gear. The rotational motion on the strings reduces the length of the strings and causes the linear motion of the load by generating pulling forces. Thus, TSA is able to transmit high forces with a very low input torque, while remaining mechanically simple. There has been much research related to the modeling and control of TSA, where TSAs were recently applied to develop tendon-driven robotic fingers [6]-[10]. Shin et al. [11] combined TSA with a passive clutch mechanism to achieve fast bending motion and large grasping force for the robot finger. Popov et al. [12] introduced a TSA model that considers a variable radius of twisted string.

The contribution of this paper is the development of impedance controlled TSA for use in tensegrity robots for reliable and robust environmental interactions. The unique properties of TSA include compact, light-weight 


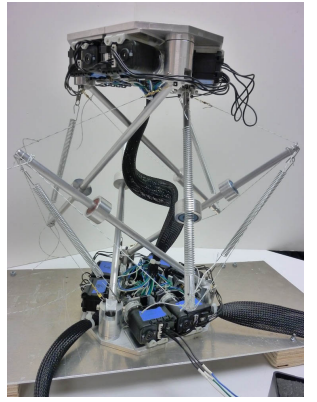

(a)TenseBot.

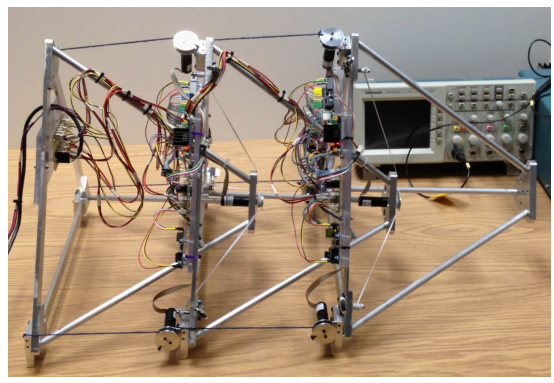

(b)TetraSpine2.

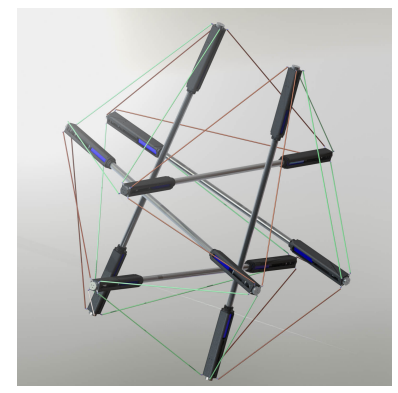

(c)SuperBallBot.

Fig. 1 Tensegrity robots in the NASA Ames Intelligent Robotics Group.

mechanical structures, inherent compliance, and variable gearing which provides an enhanced solution as an actuator of tensegrity robots. Since controllable compliance and force distribution properties are essential for tensegrity robots, impedance controlled TSAs are an ideal actuator for local control of individual strings, because they are able to adjust both a desired force trajectory and a stiffness along that trajectory. Thus, impedance control represents the ideal integration between the competing needs of position control and force control.

This paper introduces three types of tensegrity robots in the NASA Ames Intelligent Robotics Group (IRG) including its future application to planetary exploration. Next we introduce the impedance controlled TSA and an initial experimental prototype. To develop the impedance controller, we test and calibrate the position and force control behavior of the actual hardware against the theoretical performance of the system. This then allows us to implement the impedance controller, which is verified experimentally and shown to provide variable stiffness around a desired target position in the face of external disturbances. Lastly, this paper discusses the potential application of impedance controlled TSA in a closed tension system like tensegrity robots.

\section{TENSEGRITY ROBOTS}

Since 2011, the NASA Ames IRG has been actively developing tensegrity structures into robotic platforms using its control algorithm to handle unexpected contact forces and structure dynamics encountered during planetary exploration [2]-[5],[13], [14]. Tensegrity is counterintuitive tension structure with no rigid connections and is uniquely robust, light-weight, deployable, reconfigurable, and scalable [15]. Any type of applied load on the tensegrity structure is distributed as tension and compression throughout the entire structure. Thus, the tensegrity structure becomes ideal for physical interaction because it provides compliant actuation, variable stiffness, robustness to perturbations and multi-path force distribution.

\subsection{Tensegrity Robots in the NASA Ames IRG}

The NASA Ames IRG currently has two prototypes of tensegrity robots - TenseBot and TetraSpine 2 - and is building a landing and a mobility tensegrity robot called SuperBallBot for a future Titan exploration mission as shown in Fig. 1. TenseBot (Fig. 1(a)) originally created by students at Univ. of Idaho is the 6-rod tensegrity robot, which is based on the concept of a tensegrity flight simulator similar to the tensegrity-based Stewart platform [16]. However, some changes have been made to allow a wider range of motions and orientations of the upper plate in 3D space. TenseBot is made up of 6 rigid bars, 12 adjustable strings, and 6 spring elements, where the length of 12 strings is controlled by using a position controlled spooled cable actuation. Experience with this robot has shown the weakness of spooled cable actuation and the risks of strings getting tangled around the motor shaft in situations where the structure goes slack. Since TenseBot has actuation for all of the tendons as opposed to just the saddle tendons, it is used to validate the forward and inverse kinematics algorithms for tensegrity robots.

TetraSpine2 (Fig. 1(b)) is comprised of three tetrahedra, which is the simplified model of a tensegrity spine [17]. Each segment of TetraSpine2 is connected by 3 outer strings and 3 inner strings, where the string length is controlled by using an impedance controlled spool cable actuation and central pattern generator to generate the translational crawling locomotion and is based on the simulation and hardware experience of our first TetraSpine robot [3]. Due to the compliance of impedance controlled strings, TetraSpine has shown the ability to adapt to the irregular terrain and to track the reference signal given by the central pattern generator. The current research focuses on understanding the dynamics and control for integrating ground reaction forces experienced when manipulating complex terrain. 
SuperBallBot (Fig. 1(c)) is the 6-rod tensegrity icosahedron, which is capable of being compactly packed during launch, reliably unpacked at the destination, acting like a shock-absorbing air-bag during landing, and effectively deforming itself to roll to locations of scientific interest. SuperBallBot is being studied for a Titan exploration mission through a NASA Innovative Advanced Concepts (NIAC) project [2]. Performing drop tests on physical prototypes at Titan terminal velocity have confirmed that SuperBallBot is an effective landing platform [4]. In addition, robust smooth rolling motion over various terrains has been realized by evolutionary and dynamical algorithms in a physics based simulator [13],[14]. We believe that SuperBallBot can be used in future space mission with low payload cost and high reliability because it is uniquely robust, light-weight, deployable, reconfigurable, and scalable.

\section{IMPEDANCE CONTROLLED TWISTED STRING ACTUATOR}

Current NASA Ames IRG tensegrity robots are controlled by changing the length of strings, which are realized by pulling the string around a spool with a highly geared motor. However, due to these intermediate mechanisms, the spooled cable actuation causes the loss of force between actuator and string. In addition, the spooled cable actuation increases the mass of individual actuator modules, which increases the inertia of the structure and requires higher actuation power to achieve locomotion and manipulation. Finally, spooled cable actuation generally requires the motors to be mounted orthogonal to the actuated string, which can introduce mechanical design challenges. Thus, the objective of this paper is to develop an in-line alternative actuator that transforms the rotational motion to translational motion by using dynamic string twisting while satisfying robustness against external disturbances.

TSA is realized by twisting strings where a string directly connected to an actuator acts as a gear. When a load is attached to the opposite end of the string, the rotational motion on the string will reduce the length of the string and cause the translational motion of the load due to generated pulling force. Thus, TSA is able to transmit high forces with a very low input torque, while remaining mechanically simple and reliable.

Fig. 2 shows the initial prototype of TSA for a tensegrity robot, which consists of a DC motor, a pair of strings aligned along the rotation axis of the motor, a spring as the load at the other end, and a load cell to measure the force on the strings. One end of a pair of strings $(50 \mathrm{lb}$ Dacron kite string) is connected to a DC motor (MAXON 217988) for rotational motion and the other end is fixed to a load cell (Load Cell Central LCC-CTD-10) for measuring string tension. The load cell is mounted on the linear guide rail to have low sliding friction and to realize smooth movement for the TSA. Lastly, the spring (Vanel

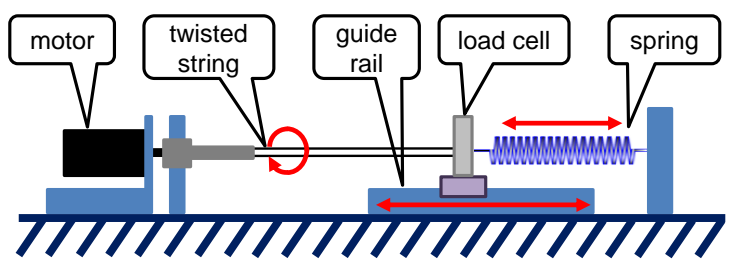

(a)Experimental representation.

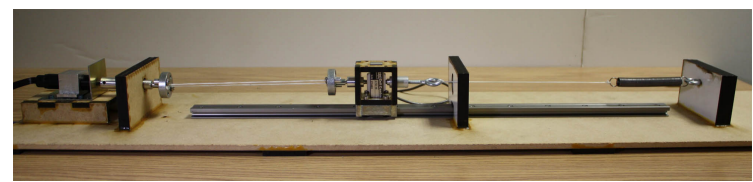

(b)Experimental platform.

Fig. 2 Experimental setup of the twisted string actuator. Table 1 Parameter settings of twisted string actuator.

\begin{tabular}{|c|c|}
\hline Parameter & Value \\
\hline \hline$L_{0}$ & $169 \mathrm{~mm}$ \\
\hline$r$ & $0.44 \mathrm{~mm}$ \\
\hline$K$ & 1.25 \\
\hline$B$ & 0.25 \\
\hline
\end{tabular}

Sarl T.100.090.0800.A) is connected to the other end of the load cell to maintain initial tension.

Since the maximum output-voltage swing of the load cell is $10 \mathrm{mV}$, the output-voltage is amplified by the instrumentation amplifier and then is connected to the analog-to-digital converter. After the calibration process, the resolution of the load cell is $0.054 \mathrm{~N}$. Similarly, the spring is calibrated to verify its characteristics, which shows great linearity once the spring is loaded at the pretension force of $2.31 \mathrm{~N}$. The resolution of the spring is $0.125 \mathrm{~N}$, which corresponds to a 1.57 percent error compared to the theoretical spring constant value of $0.127 \mathrm{~N}$.

Assuming that the pair of twisted strings can be modeled as an ideal helix and the string is rigid, the actuation length of the string at time $t$ is calculated as follows [6]:

$L_{t}=\sqrt{L_{0}^{2}-\theta^{2} r^{2}}$

where $L_{0}$ represents the initial length of twisted string, $\theta$ is the motor angle, and $r$ is the cross-section radius of twisted string. Note that the values of $L_{0}$ and $r$ for the TSA prototype are defined in Table 1.

An impedance controller is generally applied to track a combination of position based trajectories and variable stiffness when environmental dynamics are encountered[18]. Impedance control is a particularly important concept since neither force control nor position control are sufficient on their own for reliable interaction with the environment in realistic settings. An intuitive way of understanding this controller is that one can specify a trajectory to be followed, and a stiffness profile around that trajectory, such that the further the actuator is perturbed away from its desired trajectory, the stronger the restoration force it will apply to return to its desired trajectory. 
The level of stiffness and the linearity or non-linearity of that stiffness manifold can all be set according to the use case. Traditionally, impedance control has been applied to serial chain manipulators, but has also been applied to cable based actuation starting with [19], and more recently in [3], where we implemented it with a spooledcable actuator. The desired force command at time $t$ is defined as follows:

$$
F_{t}=F_{0}+K\left(L_{0}-L_{t}\right)-B v_{t}
$$

where $F_{0}$ is the initial force to maintain the string stiffness and $v_{t}$ is the actuation velocity of the string. Note that $v_{t}$ is obtained numerically based on Eq. (1). $K$ and $B$ represent the stiffness and the damping coefficient, respectively, which serve to twist or untwist the string toward the specified virtual trajectory. Note that the values of $K$ and $B$ for the TSA prototype are defined in Table 1. The advantage of using impedance control is that the TSA becomes passive and able to modify its stiffness profile such that the entire system guarantees stable interaction with all passive elements even if the external contact is applied at any point along the string.

\section{EXPERIMENTAL RESULTS}

Since impedance control is built from a combination of position and force based controls, we started by verifying and calibrating the performance of standard position and force based controllers. This is particularly important because there is no direct means of measure the absolute length of the string, yet we need string length as part of the impedance controller. While we experimented with linear stretch sensors and other means of directly measuring string length, we did not find a practical solutions for this problem, given that in a tensegrity robot the relationship between the ends of the string will not stay constant as the overall structure morphs. Thus, the string length is currently calculated by using the encoders to track rotations of the motor, and having an accurate model of the actuator behavior. Note that footage of all experimental results of TSA is available in the supplementary video.

\subsection{Position Control and Model Calibration}

Position control of TSA was implemented to verify the actual length of twisted strings compared to the theoretical model in order to be used in the impedance controller. In the position control, the motor was controlled at every $0.001 \mathrm{sec}$, where the desired motor position was sent every $0.02 \mathrm{sec}$ in real-time. The values of the load cell and motor position were fed-back at every $0.01 \mathrm{sec}$, where the resolution of motor position was 1.04 degree.

Fig. 3 shows the experimental results of both motor position and string force in a static load condition. It took $1.51 \mathrm{sec}$ to rotate 40 revolutions of TSA, where the maximum speed is set to 28.94 revolutions per second in the current configuration. The string force was increased from $2.81 \mathrm{~N}$ to $7.45 \mathrm{~N}$ at 40 revolutions, where there was

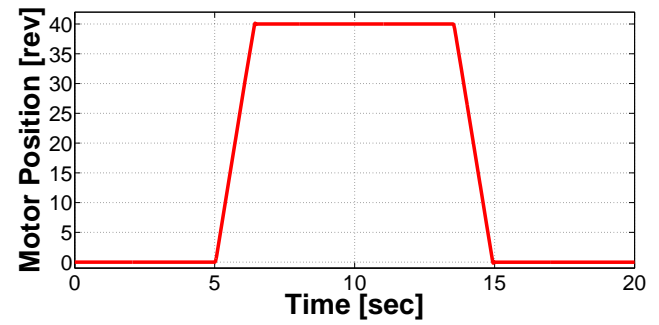

(a)Motor position.

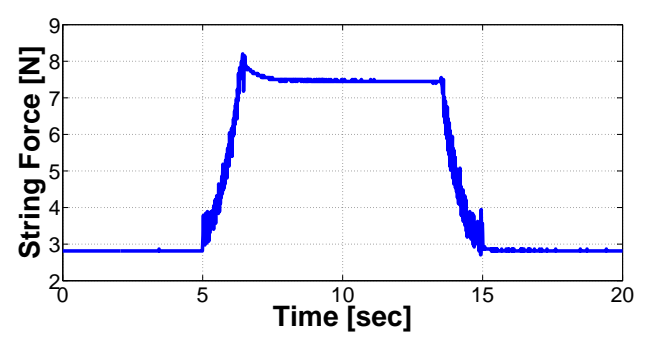

(b)String force.

Fig. 3 Rotating 40 revolutions in static load condition.

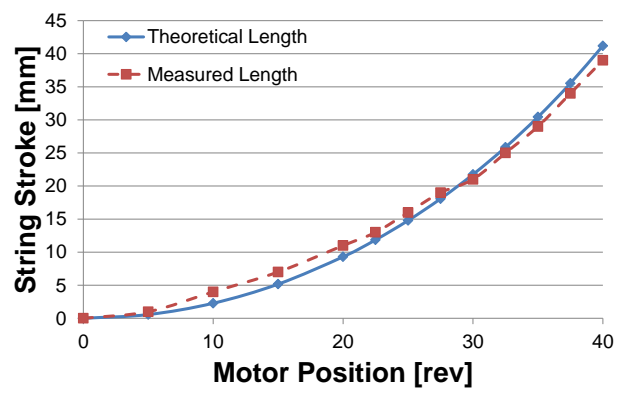

(a)String length.

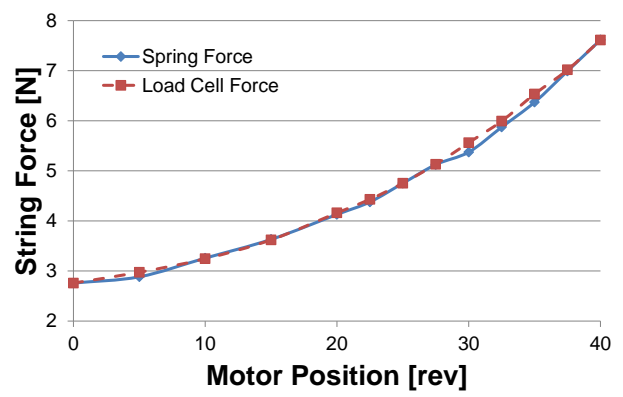

(b)String force.

Fig. 4 String length and string force in static load condition.

an overshoot of $0.75 \mathrm{~N}$ due to the controller response. The overshoot was caused by the string behavior, which was contracted more as it was twisted too fast and then released over time.

Fig. 4 shows the string length and string force in a static load condition. Fig. 4(a) shows the comparison between the theoretical stroke length of twisted string and the measured change length of spring. Note that the theoretical stroke length $\Delta L$ is equivalent to the difference between the initial length and the actuation length of spring calculated from Eq. (1). Since the cross-section radius of 


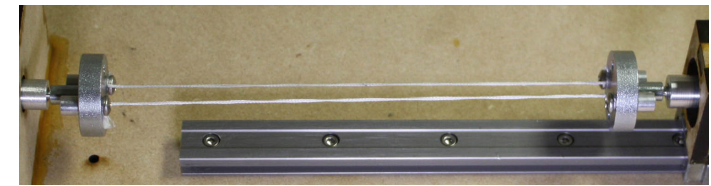

(a)Untwisted - 0 revolution.

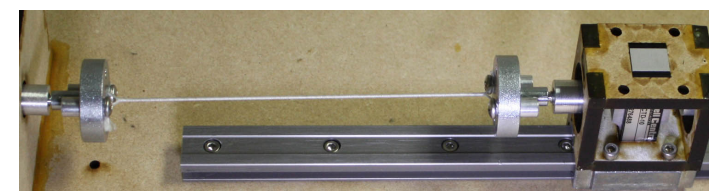

(b)Fully twisted - 40 revolutions.

Fig. 5 Snapshots of twisted string length in static load condition.

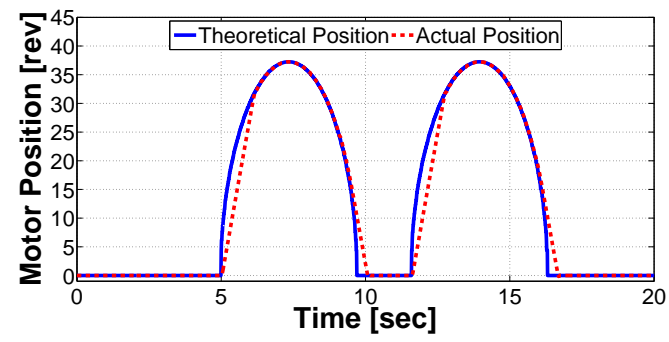

(a)Motor command and actual position.

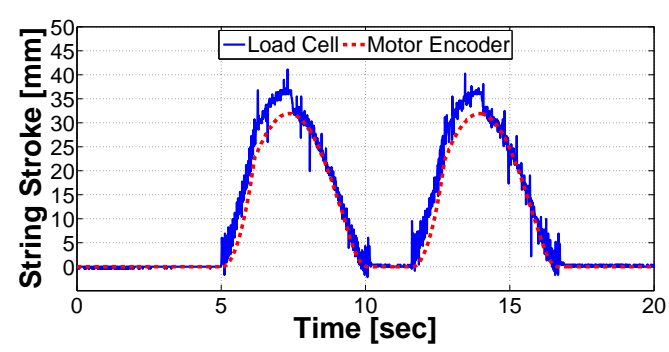

(b)Change in string length.

Fig. 6 Changing the string length in sine function using position control.

the twisted string was not identical in all the twisted region, a small error existed between the theoretical and the measured length. Fig. 4(b) shows the calculated string force using the spring length and load cell measurement. The string force error between two measurements is minimized due to the proper calibration procedure. Fig. 5 shows the snapshots of twisted string in two states: untwisted and fully twisted state.

Since most locomotion gaits for a tensegrity robot involve rhythmic oscillations in the structure with phaseoffset controls in different actuators, we also tested the behavior of the TSA when following a sine function in string stroke length, which was implemented as,

$\Delta L_{t}=\left|35.0 \sin \left(\frac{\pi}{5.0} t\right)\right|$.

Fig. 6 shows the motor command and the corresponding string stroke length. Fig. 6(a) shows the comparison between the theoretical motor position calculated using Eq. (1) and the actual motor position measured using the encoder feedback signals. The maximum speed of the motor was unable to follow the theoretical motor position because the TSA required a large number of rotations to decrease the string stroke length in the early stage of twisted string. However, this could be solved by increasing the period of sine wave.

Fig. 6(b) represents the calculated string stroke length using the load cell and motor encoder signals. The magnitude of both signals showed that the string stroke length was changing in sine waveform with the frequency of 5.0 sec. In order to calculate the string stroke length using the load cell value, it was converted as a string force, and then applied the spring constant. Since the load cell force and spring force were not identical, the calculated maximum amplitude of stroke length using the load cell was $36.37 \mathrm{~mm}$, which corresponded to 3.91 percentage error. The motor encoder signal and Eq. (1) were used to calculate the stroke length. Similarly, due to the model error, the calculated maximum amplitude was $31.91 \mathrm{~mm}$, which corresponded to 8.83 percentage error. Consequently, it is difficult to control the exact length of string unless a position potentiometer or stretch sensor is attached in parallel to the TSA.

\subsection{Force Control and Impedance Control}

The proposed TSA was verified with force control and impedance control to maintain a sufficient amount of force on the string in dynamic environment. Due to the sensitivity of load cell value, the motor was controlled at every $0.00025 \mathrm{sec}$. Note that if the values of $K$ and $B$ in Eq. (2) were set to zero, then it simply reduced to the force control.

Fig. 7 shows the string force and the motor position using force control and impedance control. The desired force command in force control was to maintain $5 \mathrm{~N}$ of string force, whereas the objective of impedance control was to maintain $5 \mathrm{~N}$ of string force plus the deflection dependent force. External contact force was applied by pushing the load cell module in both directions. As shown in Fig. 7(a), the force control either twisted or untwisted the strings to always maintain $5 \mathrm{~N}$ of desired string force. In contrast, the impedance control either twisted or untwisted the strings by modifying the desired string force value to match the impedance of contact force as shown in Fig. 7(b). Note that the impedance controlled TSA generated a higher magnitude of force as the displacement of the twisted springs moved further away from the target position.

Fig. 8 shows the results of force control following a sine function. Initially, the TSA was set as the initial configuration and the system power was turned on at $4 \mathrm{sec}$. The spring was grabbed by a hand from $18 \mathrm{sec}$ to $30 \mathrm{sec}$, which was applied as an external disturbance. As shown in Fig. 8, the force trajectory successfully followed the sine function with the presence of external disturbance by adjusting the motor position. 
Force Control
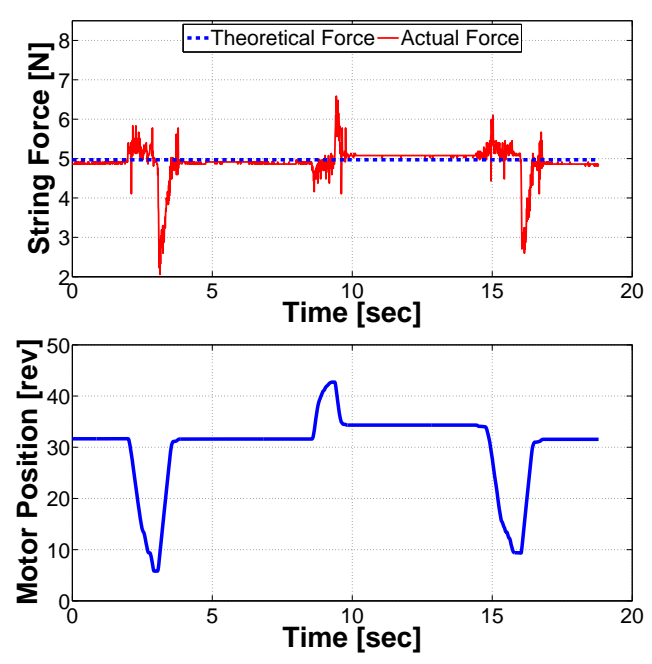

(a)Maintaining $5 \mathrm{~N}$ of string force using force control
Impedance Control
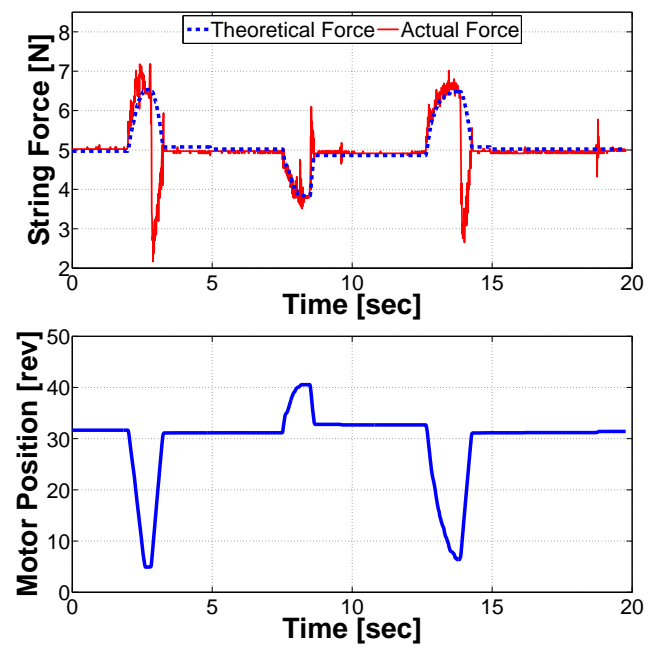

(b)Deflection dependent control using impedance control

Fig. 7 Comparison between force control and impedance control with the presence of external disturbance.

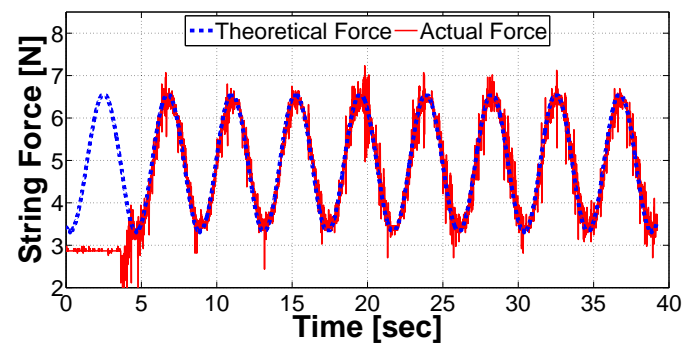

(a)Change in string force.

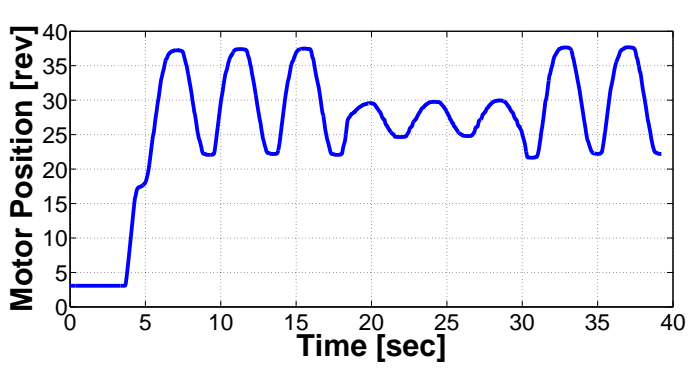

(b)Motor position.

Fig. 8 Changing the string force in sine function using force control with the presence of external disturbance.

Significantly, the proposed impedance controlled TSA increased the stiffness of system by increasing the desired force command when the contact force was applied to the direction of untwisting strings and vice versa. When the values of $K$ and $B$ are properly defined as functions of normalized string tension and normalized twisted string lengths, the impedance controlled TSA can control the actuator compliance, preventing the strings from going slack, and providing robust environmental interactions.

\section{DISCUSSION AND FUTURE WORK}

While we found some limitations to the accuracy of pure position control of the TSA, we were still able to achieve reasonable performance under force and impedance control. This is because the strings, when twisted, act somewhat like a spring and are naturally compliant, making them stretch under load and distort from the exact desired position. Yet pure position control is rarely relevant for robots which interact with the natural world, rather it is force and impedance controls which are the dominant needs. While the twisted strings might be slightly compliant, the experienced forces are accurately reflected and controlled. Thus, we see that the physical properties of the TSA match the properties of impedance control - being compliant around a desired target position. Since tensegrity robots are also structurally compliant, it is pointless to be overly demanding of exact position control. Thus, while TSA's inherent compliance may make them unsuitable for some applications, it appears that they are a natural match to tensegrity robots.

Given our initial success with this approach, looking to the future we plan to formally compare the performance difference between TSA and spooled cable actuators. While our experience indicated, and the literature often claims, that TSA can provide high torque at a lower system mass than traditional spooled cable actuation, we intend to make a direct evaluation of these claims. By remounting the exact same motor in two different orientations such that we can either drive the TSA or attach a spool, we will be able to measure the differences in torque production, energy efficiency, and other intrinsic qualities between the two approaches. To our knowledge, such a direct experimental comparison has never been reported in the literature. 
One of the challenges of tensegrity robots is that as they deform and change shapes, the angles at which the strings attach to the rods changes. This can cause problems for spooled cable actuators as the strings are no longer winding straight onto the spools, but rather may be rubbing on the side walls of the spools and increasing the chances that they will be pulled off and start wrapping around the motor axle. We believe that this is another advantage of TSA - that they should perform well even if the load is not pulling directly inline with the motor. We will thus perform experiments where we vary the position of the loaded end of the string relative to the motor to evaluate TSA performance under these conditions.

Finally, and more speculatively, we note that, much like animal physiology, tensegrity strings are fundamentally in antagonistic formations. In most motions, some strings will be lengthening while other strings are shortening. Because of the variable gearing of TSA, a "short" string will be highly twisted, and thus will have high gearing and will consequently be "stronger". On the other hand, a long string has fewer twists, and it thus has lower gearing, and is thus "weaker". We suspect that this is advantageous as it helps avoid antagonistic fighting between the actuators. If the desire is to shift shape such that a long string shortens, using TSA this will only be possible if the antagonistic "short" string relaxes and unwinds, as there is little that the low-geared long string can do to overpower it. In comparison, if the strings had equal gearing, and thus power, the long string could start shortening and fighting the short string, leading to chatter and instability in the system. Thus, the asymmetrical gearing of TSA lends a physical stability to control when deployed in antagonistic architectures. This enables looser coupling and coordination between controllers, which is helpful in tensegrity robots which are often implemented in a distributed modular manner. While this concept is currently speculative, we look forward to investigating it in the future.

\section{ACKNOWLEDGMENT}

This research was supported by an appointment to the NASA Postdoctoral Program at the Ames Research Center, and administered by Oak Ridge Associated Universities through a contract with NASA. Support was also provided by NASA's Human Robotic Systems (HRS) project, Game Changing Developments (GCD) Program, Space Technology Mission Directorate (STMD).

The authors would like to thank Jonathan Bruce, Andrew Sabelhaus, George Korbel, Ken Caluwaerts, Brian Tietz, Jeffrey Friesen, Alexandra Pogue, Terry Fong, and the NASA Ames Intelligent Robotics Group.

\section{REFERENCES}

[1] G. Tibert, "Deployable tensegrity structures for space applications," Ph.D. Dissertation, Royal Inst.
Technol. (KTH), 2002.

[2] A. Agogino, V. SunSpiral and D. Atkinson, Super Ball Bot - Structures for planetary landing and exploration, NASA Innovative Advanced Concepts Program, Final Report, 2013.

[3] B. Tietz, R. Carnahan, R. Bachmann, R. Quinn and V. SunSpiral, "Tetraspine: Robust terrain handling on a tensegrity robot using central pattern generators," Proc. of IEEE/ASME Int. Conf. Advanced Intelligent Mechatronics, pp. 261-267, 2013.

[4] V. SunSpiral, G. Gorospe, J. Bruce, A. Iscen, G. Korbel, S. Milam, A. Agogino and D. Atkinson, "Tensegrity based probes for planetary exploration: Entry, descent and landing (EDL) and surface mobility analysis," Proc. 10th Int. Planetary Probe Workshop, 2013.

[5] K. Caluwaertz, J. Despraz, A. Iscen, A. Sabelhaus, J. Bruce and V. SunSpiral, "Design and control of compliant tensegrity robots through simulation and hardware validation," J. Roy. Soc. Interface, Vol. 11, No. 98, 2014.

[6] T. Wurtz, C. May, B. Holz, C. Natale, G. Palli and C. Melchiorri, "The twisted string actuation system: modeling and control," Proc. of IEEE/ASME Int. Conf. Advanced Intelligent Mechatronics, pp. 1215-1220, 2010.

[7] G. Palli, G. Borghesan and C. Melchiorri, "Modeling, identification, and control of tendon-based actuation systems," IEEE Trans. Robot., Vol. 28, No. 2, pp. 277-290, 2012.

[8] G. Palli, C. Natale, C. May, C. Melchiorri and T. Wurtz, "Modeling and control of the twisted string actuation system," IEEE/ASME Trans. Mechatronics, Vol. 18, No. 2, pp. 664-673, 2013.

[9] T. Sonoda and I. Godler, "Position and force control of a robotic finger with twisted strings actuation," Proc. of IEEE/ASME Int. Conf. Advanced Intelligent Mechatronics, pp. 611-616, 2011.

[10] T. Sonoda, K. Ishii, A. Nassiraei and I. Godler, "Control of robotic joint by using antagonistic pair of twist drive actuators," Proc. of 38th Annual Conf. IEEE Ind. Electron. Soc., pp. 5410-5415, 2012.

[11] Y. Shin, H. Lee, K.-S. Kim and S. Kim, "A robot finger design using a dual-mode twisting mechanism to achieve high-speed motion and large grasping force," IEEE Trans. Robot., Vol. 28, No. 6, pp. 1398-1405, 2012.

[12] D. Popov, I. Gaponov and J.-H. Ryu, "A study on twisted string actuation systems: mathematical model and its experimental evaluation," Proc. of IEEE/RSJ Int. Conf. Intell. Robots Syst., pp. 12451250, 2012.

[13] A. Iscen, A. Agogino, V. SunSpiral and K. Tumer, "Controlling tensegrity robots through evolution," Proc. Genetic and Evol. Comput. Conf., pp. 12931300, 2013. 
[14] A. Iscen, A. Agogino, V. SunSpiral and K. Tumer, "Learning to control complex tensegrity robots," Proc. 12th Int. Conf. Autonomous Agents and Multiagent Syst., pp. 1193-1194, 2013.

[15] R. Skelton, J. Helton, R. Adhikari, J. Pinaud and W. Chan, An Introduction to the Mechanics of Tensegrity Structures, CRC Press, 2002.

[16] C. Sultan, M. Corless and R. Skelton, "Tensegrity flight simulator," J. Guidance, Control, and Dynamic, Vol. 23, No. 6, pp. 1055-1064, 2000.

[17] S. Leven, "The tensegrity-truss as a model for spine mechanics: Biotensegrity," J. Mech. Med. Biol., Vol. 2, pp. 375-388, 2002.

[18] N. Hogan, "Impedance control: An approach to manipulation: Part I-III," J. of Dynamic Syst. Meas. Control, Vol. 107, pp. 1-24, 1985.

[19] O. Orki, O. Shai, A. Ayali and U. Ben-Hanan, "A model of caterpillar locomotion based on assur tensegrity structures," Proc. of ASME Int. Design Eng. Tech. Conf. \& Comput. and Inform. in Eng., pp. 1-7, 2011. 